\title{
Polarized proton-deuteron Drell-Yan processes and parton distributions
}

\author{
S. Hino and S. Kumano * \\ Department of Physics \\ Saga University \\ Saga 840-8502, Japan
}

\author{
Talk at the KEK-Tanashi International Symposium \\ on PHYSICS OF HADRONS AND NUCLEI \\ Tokyo, Japan, Dec. $14-17,1998$ \\ (talk on Dec. 16, 1998)
}

* Email:97sm16@edu.cc.saga-u.ac.jp, kumanos@cc.saga-u.ac.jp

Information on their research is available at http://www2.cc.saga-u.ac.jp/saga-u/riko /physics/quantum1/structure.html. 


\title{
Polarized proton-deuteron Drell-Yan processes and parton distributions
}

\author{
S. Hino and S. Kumano ${ }^{\text {a }}$ * \\ ${ }^{a}$ Department of Physics, Saga University, Saga 840-8502, Japan
}

We show in general that there are 108 structure functions in the proton-deuteron DrellYan processes. However, there exist only 22 finite ones after integrating the cross section over the virtual-photon transverse momentum $\vec{Q}_{T}$ or after taking the limit $Q_{T} \rightarrow 0$. There are 11 new structure functions in comparison with the ones of the proton-proton reactions, and they are related to the tensor structure of the deuteron. Parton-model analyses indicate an important tensor structure function $V_{T}^{U Q_{0}}$, which can be measured by a quadrupole spin asymmetry. The Drell-Yan process has an advantage over lepton reactions in finding tensor polarized antiquark distributions. We hope that our studies will be realized in the next-generation RHIC-Spin project and other ones.

\section{Introduction}

Spin structure of the proton has been investigated through polarized deep inelastic lepton scattering. It is now interesting to investigate other aspects of spin physics. For example, the deuteron is a spin-one particle, so that it has tensor structure which does not exist in the proton. The deuteron target is often used in the deep inelastic scattering; however, the purpose is to extract the "neutron" structure functions in the deuteron. We had better shed light on the deuteron spin structure itself rather than just use it for finding the neutron information. There are some initial studies on the spin-one structure functions. In the lepton scattering on the deuteron, there exist new structure functions, $b_{1}, b_{2}, b_{3}$, and $b_{4}$. However, the polarized proton-deuteron (pd) reaction had not been studied at all in connection with the deuteron structure functions. The major purpose of this work is to investigate the general formalism of the polarized pd Drell-Yan processes [1]. In particular, we list all the possible structure functions in the reactions. Then, the processes are also analyzed in a naive parton model [2]. Another purpose is to facilitate future deuteron projects such as the polarized deuteron reactions at RHIC.

The tensor structure function is a new field of high-energy spin physics. Because it has not been measured experimentally, it is a unique opportunity to test our present understanding of spin physics. Furthermore, it may reveal an unexpected aspect of hadron structure. There is a possibility that the polarized proton-deuteron reaction is realized in the future experimental projects.

\footnotetext{
*http://www2.cc.saga-u.ac.jp/saga-u/riko/physics/quantum1/structure.html. S.K. was partly supported by the Grant-in-Aid for Scientific Research from the Japanese Ministry of Education, Science, and Culture under the contract number 10640277.
} 


\section{Structure functions and spin asymmetries}

Because the polarized high-energy pd Drell-Yan process had not been studied, we should first discuss in general what kind of structure functions are investigated [1]. Two independent formalisms are studied. The first one is a spin-density formalism and the second is a hadron-tensor formalism. In the first method, we express structure functions by the helicity amplitudes and Clebsch-Gordan coefficients. Then, imposing Hermiticity, parity conservation, and time-reversal invariance, we find that 108 structure functions exist. In comparison with the proton-proton (pp) reactions, there are 60 new functions. If the cross section is integrated over the lepton-pair transverse momentum $\vec{Q}_{T}$, there are only 22 ones and the others vanish. There are 11 new functions in addition to the ones of the pp reactions, and they are associated with the tensor structure of the deuteron. In the second method, possible Lorentz index expressions are found for the hadron tensor $W^{\mu \nu}$ by the combinations of available Lorentz vectors and pseudovectors. The $Q_{T} \rightarrow 0$ case indicates that the following 22 structure functions also exist:

$$
\begin{aligned}
& W_{0,0}, \quad V_{0,0}^{L L}, \quad V_{0,0}^{T T}, \quad V_{0,0}^{U Q_{0}}, \quad V_{0,0}^{T Q_{1}}, W_{2,0}, \quad V_{2,0}^{L L}, \quad V_{2,0}^{T T}, \quad V_{2,0}^{U Q_{0}}, V_{2,0}^{T Q_{1}}, U_{2,1}^{T U}, \\
& U_{2,1}^{T Q_{0}}, \quad U_{2,1}^{U T}, \quad U_{2,1}^{L Q_{1}}, U_{2,1}^{T Q_{2}}, U_{2,1}^{T L}, \quad U_{2,1}^{L T}, U_{2,1}^{U Q_{1}}, U_{2,2}^{U Q_{2}}, U_{2,2}^{T T}, U_{2,2}^{T Q_{1}}, U_{2,2}^{L Q_{2}} \text {. }
\end{aligned}
$$

The functions $W, V$, and $U$ are an unpolarized structure function, a polarized one without the spin factors in the hadron tensor, and a polarized one with the spin factor. The subscripts of these structure functions indicate, for example, that $W_{L, M}$ is obtained by $\int d \Omega Y_{L M} d \sigma /\left(d^{4} Q d \Omega\right) \propto W_{L, M}$ in the unpolarized reaction. The superscripts indicate the polarization states of $A$ and $B$ : e.g. $U_{L, M}^{p o l_{A}}$ pol $_{B}$. The superscripts $U, L$, and $T$ show unpolarized, longitudinally polarized, and transversely polarized states. The quadrupole polarizations $Q_{0}, Q_{1}$, and $Q_{2}$ are specific in the reactions with a spin-1 hadron, and they are associated with the spherical harmonics $Y_{20}, Y_{21}$, and $Y_{22}$ as shown in Figs. 1, 2, and 3. They are the polarizations in the $x z, y z$, and $x y$ planes. The 11 structure functions with the superscripts $Q_{0}, Q_{1}$, and $Q_{2}$ do not exist in the pp reactions.

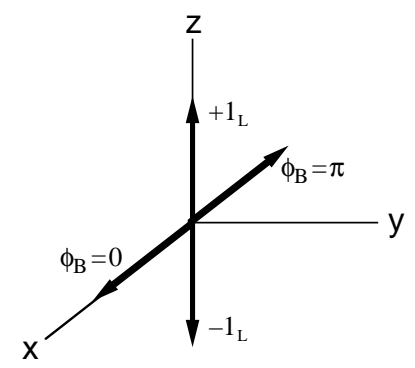

Figure 1. Polarization $Q_{0}$.

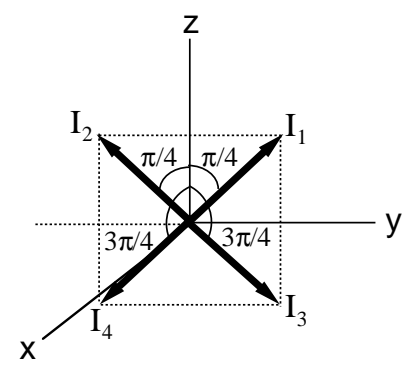

Figure 2. Polarization $Q_{1}$.

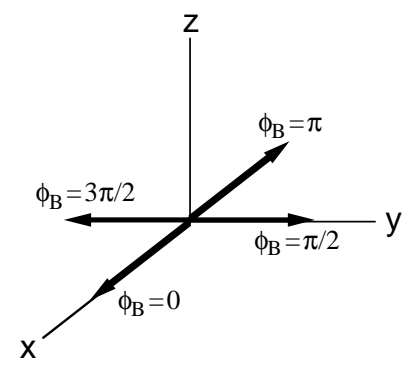

Figure 3. Polarization $Q_{2}$.

In the pp Drell-Yan processes, merely the following unpolarized, longitudinal, and transverse combinations exist: $\langle\sigma\rangle, A_{L L}, A_{T T}, A_{L T}$, and $A_{T}$. In addition, the quadrupole spin asymmetries exist in the pd reactions. Our formalism indicates that the following 
fifteen quantities could be investigated:

$$
\begin{aligned}
& <\sigma>, \quad A_{L L}, \quad A_{T T}, \quad A_{L T}, \quad A_{T L}, \quad A_{U T}, \quad A_{T U} \\
& A_{U Q_{0}}, A_{T Q_{0}}, A_{U Q_{1}}, A_{L Q_{1}}, A_{T Q_{1}}, A_{U Q_{2}}, A_{L Q_{2}}, A_{T Q_{2}} \text {, }
\end{aligned}
$$

where the subscript $U$ indicates the unpolarized state. These asymmetries are expressed in terms of the structure functions in Eq. (1). For example, $A_{U Q_{0}}$ is measured with the unpolarized proton and the $Q_{0}$-type tensor polarized deuteron. It is written by the structure functions $V_{0,0}^{U Q_{0}}, V_{2,0}^{U Q_{0}}, W_{0,0}$, and $W_{2,0}$ :

$A_{U Q_{0}}=\frac{1}{2<\sigma>}\left[\sigma\left(\bullet, 0_{L}\right)-\frac{\sigma\left(\bullet,+1_{L}\right)+\sigma\left(\bullet,-1_{L}\right)}{2}\right]=\frac{2 V_{0,0}^{U Q_{0}}+\left(\frac{1}{3}-\cos ^{2} \theta\right) V_{2,0}^{U Q_{0}}}{2 W_{0,0}+\left(\frac{1}{3}-\cos ^{2} \theta\right) W_{2,0}}$

where $\bullet$ indicates the unpolarized case.

\section{Parton-model analysis}

In the previous section, it is shown in Eq. (1) that there exist the 22 structure functions. These functions are measured by the spin asymmetries in Eq. (2). Next, we try to understand the physics meaning of these structure functions, particularly the new tensor polarized ones, in a naive parton model [2].

The hadron tensor $W^{\mu \nu}$ is written by the quark and antiquark correlation functions. They are expressed in terms of possible combinations of Lorentz vectors and pseudovectors with the constrains of Hermiticity, parity conservation, and time-reversal invariance. We find that new tensor distributions are involved in the correlation function $\Phi\left[\gamma^{+}\right]$. It is particularly important that the leading-twist tensor distribution $\delta q$ is found in our analysis, and it is expressed by the "unpolarized" quark distribution $\left[\mathcal{P}(x)_{\lambda}\right]$ in a spin-1 hadron with helicity $\lambda$ as

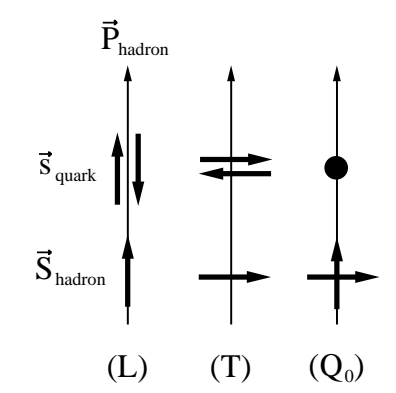

$$
\delta q(x)=\frac{1}{2}\left[\mathcal{P}(x)_{\lambda=0}-\frac{\mathcal{P}(x)_{\lambda=+1}+\mathcal{P}(x)_{\lambda=-1}}{2}\right] .
$$

It agrees with the definition in the lepton-deuteron scattering [3]. In the naive partonmodel analysis, we find 19 structure functions. However, there exist only four finite structure functions by the $\vec{Q}_{T}$ integration and the others vanish. In the following, the $\vec{Q}_{T}$ integrated results are shown. First, the cross section is

$$
\begin{gathered}
\frac{d \sigma}{d x_{A} d x_{B} d \Omega}=\frac{\alpha^{2}}{4 Q^{2}}[ \\
\left(1+\cos ^{2} \theta\right)\left\{\bar{W}_{T}+\frac{1}{4} \lambda_{A} \lambda_{B} \bar{V}_{T}^{L L}+\frac{2}{3}\left(2\left|\vec{S}_{B T}\right|^{2}-\lambda_{B}^{2}\right) \bar{V}_{T}^{U Q_{0}}\right\} \\
\left.+\sin ^{2} \theta\left|\vec{S}_{A T}\right|\left|\vec{S}_{B T}\right| \cos \left(2 \phi-\phi_{A}-\phi_{B}\right) \bar{U}_{2,2}^{T T}\right]
\end{gathered}
$$


where $\bar{W}=\int d^{2} \vec{Q}_{T} W$ and the same for $\bar{V}$ and $\bar{U}$. The four finite structure functions are expressed by the parton distributions in the process $q$ (in $\mathrm{p})+\bar{q}($ in $\mathrm{d}) \rightarrow \ell^{+}+\ell^{-}$as

$$
\begin{aligned}
\bar{W}_{T} & =\frac{1}{3} \sum_{a} e_{a}^{2} q_{a}\left(x_{A}\right) \bar{q}_{a}\left(x_{B}\right), & \bar{V}_{T}^{L L} & =-\frac{4}{3} \sum_{a} e_{a}^{2} \Delta q_{a}\left(x_{A}\right) \Delta \bar{q}_{a}\left(x_{B}\right), \\
\bar{U}_{2,2}^{T T} & =\frac{1}{3} \sum_{a} e_{a}^{2} \Delta_{T} q_{a}\left(x_{A}\right) \Delta_{T} \bar{q}_{a}\left(x_{B}\right), & \bar{V}_{T}^{U Q_{0}} & =\frac{1}{3} \sum_{a} e_{a}^{2} q_{a}\left(x_{A}\right) \delta \bar{q}_{a}\left(x_{B}\right) .
\end{aligned}
$$

The unpolarized, longitudinally-polarized, transversity, and tensor-polarized distributions are expressed by $q_{a}, \Delta q_{a}, \Delta_{T} q_{a}$, and $\delta q_{a}$ with the quark flavor $a$, respectively. The tensor distribution can be investigated by the unpolarized-quadrupole $Q_{0}$ asymmetry

$$
A_{U Q_{0}}=\frac{\bar{V}_{T}^{U Q_{0}}}{\bar{W}_{T}}=\frac{\sum_{a} e_{a}^{2}\left[q_{a}\left(x_{A}\right) \delta \bar{q}_{a}\left(x_{B}\right)+\bar{q}_{a}\left(x_{A}\right) \delta q_{a}\left(x_{B}\right)\right]}{\sum_{a} e_{a}^{2}\left[q_{a}\left(x_{A}\right) \bar{q}_{a}\left(x_{B}\right)+\bar{q}_{a}\left(x_{A}\right) q_{a}\left(x_{B}\right)\right]},
$$

where the contribution from the process $\bar{q}($ in $\mathrm{A})+q($ in $\mathrm{B}) \rightarrow \ell^{+}+\ell^{-}$is added. This equation suggests that the tensor polarized distributions are found by the Drell-Yan experiment if the unpolarized distributions are well known in the proton. In the large $x_{F}$ region, the term $\bar{q}_{a}\left(x_{A}\right) \delta q_{a}\left(x_{B}\right)$ is neglected so that the antiquark tensor polarization $\delta \bar{q}$ could be extracted. It could be also found in the lepton scattering by studying a deviation from the $b_{1}$ sum rule [4]; however, the Drell-Yan process has an advantage over the lepton reactions in the sense that the antiquark distributions $\delta q_{a}(x)$ are found rather easily. In addition, the antiquark flavor asymmetries $\Delta \bar{u} / \Delta \bar{d}$ and $\Delta_{T} \bar{u} / \Delta_{T} \bar{d}$ can be investigated by combining the pd Drell-Yan data with the pp data in the same way as the unpolarized case [5] because the polarized pd Drell-Yan formalism is now completed in Refs. [1,2].

\section{Summary}

We have found that there exist 108 structure functions in the pd Drell-Yan processes; however, the number becomes 22 after integrating the cross section over the transverse momentum $\vec{Q}_{T}$ or after taking the limit $Q_{T} \rightarrow 0$. The new structure functions are associated with the tensor structure of the deuteron. The naive parton-model analyses

indicate the important structure function $V_{T}^{U Q_{0}}$, which is expressed by the unpolarized distributions of the proton and the tensor polarized ones of the deuteron. The pd DrellYan is particularly useful for finding the tensor polarized antiquark distributions. There are a variety of interesting topics on the polarized pd reactions. We hope that our studies will be realized experimentally.

\section{REFERENCES}

1. S. Hino and S. Kumano, SAGA-HE-136-98 (hep-ph/9810425), Phys. Rev. D in press.

2. S. Hino and S. Kumano, SAGA-HE-139-98 (hep-ph/9902258).

3. S. Kumano, pp. 371 in THE ELFE PROJECT, edited by J. Arvieux and E. De Sanctis, Italian Physical Society, Conference Proceedings Vol. 44 (1993).

4. F. E. Close and S. Kumano, Phys. Rev. D42 (1990) 2377.

5. S. Kumano, Phys. Rep. 303 (1998) 183. 